\title{
Molecular analysis of type 3 fimbrial genes from Escherichia coli, Klebsiella and Citrobacter species
}

\author{
Cheryl-lynn Y Ong ${ }^{1}$, Scott A Beatson ${ }^{1}$, Makrina Totsika' ${ }^{1}$, Christiane Forestier ${ }^{2}$, Alastair G McEwan ${ }^{1}$ and \\ Mark A Schembri*1
}

\begin{abstract}
Background: Catheter-associated urinary tract infection (CAUTI) is the most common nosocomial infection in the United States and is caused by a range of uropathogens. Biofilm formation by uropathogens that cause CAUTI is often mediated by cell surface structures such as fimbriae. In this study, we characterised the genes encoding type 3 fimbriae from CAUTI strains of Escherichia coli, Klebsiella pneumoniae, Klebsiella oxytoca, Citrobacter koseri and Citrobacter freundii.

Results: Phylogenetic analysis of the type 3 fimbrial genes ( $m r k A B C D$ ) from 39 strains revealed they clustered into five distinct clades (A-E) ranging from one to twenty-three members. The majority of sequences grouped in clade $A$, which was represented by the mrk gene cluster from the genome sequenced K. pneumoniae MGH78578. The E. coli and K. pneumoniae mrkABCD gene sequences clustered together in two distinct clades, supporting previous evidence for the occurrence of inter-genera lateral gene transfer. All of the strains examined caused type 3 fimbriae mediated agglutination of tannic acid treated human erythrocytes despite sequence variation in the mrkD-encoding adhesin gene. Type 3 fimbriae deletion mutants were constructed in 13 representative strains and were used to demonstrate a direct role for type 3 fimbriae in biofilm formation.

Conclusions: The expression of functional type 3 fimbriae is common to many Gram-negative pathogens that cause CAUTI and is strongly associated with biofilm growth. Our data provides additional evidence for the spread of type 3 fimbrial genes by lateral gene transfer. Further work is now required to substantiate the clade structure reported here by examining more strains as well as other bacterial genera that make type 3 fimbriae and cause CAUTI.
\end{abstract}

\section{Background}

Catheter-associated urinary tract infection (CAUTI) is the most common nosocomial infection in the United States and a frequent cause of bacteremia [1]. Nosocomial CAUTI is caused by a range of different bacterial pathogens [2] and these are often resistant to multiple antibiotics [3].

Biofilm formation is a trait commonly found among CAUTI isolates and results in the growth of bacteria on the inner surface of the urinary catheter. Biofilm formation promotes encrustation and protects the bacteria from the hydrodynamic forces of urine flow, host defenses and antibiotics [4]. A perquisite to biofilm growth is adherence to the catheter surface. A number of mechanisms by which Gram-negative pathogens mediate

* Correspondence: m.schembri@uq.edu.au

${ }^{1}$ Centre for Infectious Disease Research, School of Chemistry and Molecular Biosciences, University of Queensland, Brisbane, Queensland 4072, Australia Full list of author information is available at the end of the article adherence to biotic and abiotic surfaces have been described and include fimbriae (e.g. type 1, type 3, type IV, curli and conjugative pili), cell surface adhesins (e.g. autotransporter proteins such as antigen 43, UpaH and UpaG) and flagella [5-16].

The expression of type 3 fimbriae has been described from many Gram-negative pathogens [17-28]. Type 3 fimbriae are 2-4 $\mathrm{nm}$ wide and 0.5-2 $\mu \mathrm{m}$ long surface organelles that are characterised by their ability to mediate agglutination of tannic acid-treated human RBC (MR/ $K$ agglutination) [29]. Several studies have clearly demonstrated a role for type 3 fimbriae in biofilm formation [17,28,30-33]. Type 3 fimbriae also mediate various adherence functions such as binding to epithelial cells (from the respiratory and urinary tracts) and extracellular matrix proteins (e.g. collagen V) [31,34-36].

Type 3 fimbriae belong to the chaperone-usher class of fimbriae and are encoded by five genes ( $m r k A B C D F)$ arranged in the same transcriptional orientation $[29,37]$. 
The $m r k$ gene cluster is similar to other fimbrial operons of the chaperone-usher class in that it contains genes encoding major $(m r k A)$ and minor $(m r k F)$ subunit proteins as well as chaperone- $(m r k B)$, usher- $(m r k C)$ and adhesin- $(m r k D)$ encoding genes [37,38]. A putative regulatory gene $(m r k E)$ located upstream of $m r k A$ has been described previously in Klebsiella pneumoniae [37]. The mrk genes have been shown to reside at multiple genomic locations, including the chromosome [39], on conjugative plasmids $[17,30]$ and within a composite transposon [40]. Transfer of an mrk-containing conjugative plasmid to strains of Salmonella enterica serovar Typhimurium, Klebsiella pneumoniae, Enterobacter aerogenes and Kluyvera species has also been demonstrated [17]. Taken together, these data strongly support spread of the $m r k$ genes between Gram-negative pathogens by lateral gene transfer.

Recently, we identified and characterised the role of type 3 fimbriae in biofilm formation from an Escherichia coli strain isolated from a patient with CAUTI [28]. We also demonstrated that the $m r k B$ chaperone-encoding gene and the ability to mediate MR/K agglutination was common in uropathogenic Klebsiella pneumoniae, Klebsiella oxytoca and Citrobacter koseri strains (86.7\%, 100\% and $100 \%$ of strains, respectively) but rare in uropathogenic E. coli and Citrobacter freundii strains $(3.2 \%$ and $14.3 \%$ of strains, respectively) [28]. Despite the occurrence of type 3 fimbriae genes among a range of different Gram-negative bacteria that cause CAUTI, little is known about their molecular relationship. In this study, we have examined the phylogenetic correlation between type 3 fimbrial ( $m r k$ ) genes from 33 CAUTI strains representing five different uropathogens (E. coli, K. pneumoniae, K. oxytoca, C. koseri and C. freundii). We also demonstrate functional expression of type 3 fimbriae in each of these strains and describe a common role for type 3 fimbriae in biofilm formation.

\section{Results \\ Phylogenetic analysis of the mrkABCD genes from uropathogenic bacterial genera}

To investigate the phylogenetic relationship of the $m r k$ genes from 33 CAUTI strains (representing E. coli, $K$. pneumoniae, $K$. oxytoca, C. koseri and C. freundii) we amplified and sequenced an internal segment of the $m r k A, m r k B, m r k C$ and $m r k D$ genes from each strain. We also examined the corresponding sequence from six additional $m r k$ gene clusters available at GenBank. A majority-rule consensus maximum likelihood (ML) tree was constructed from the 39 concatenated mrkABCD fragments. The phylogenetic analysis indicated that the sequences clustered into five major clades (referred to as clade A to E) with good bootstrap support (Fig. 1). The five clades range from one member (clade $\mathrm{C}$, represented by $C$. freundii M46) to 23 members (clade $\mathrm{A}$, represented by $K$. pneumoniae MGH78578), with an average interallelic diversity of $11.2 \%$. Whereas the 10 C. koseri sequences clustered in a single clade (clade E), clade B (3 sequences) and clade A (23 sequences) consist of sequences from both $K$. pneumoniae and E. coli. Phylogenetic analysis using parsimony or distance-based methods produced tree topologies very similar to those obtained by using DNA maximum likelihood (data not shown).

The incongruence between the mrk consensus tree and the established phylogeny for enteric bacteria [41] is prima facie evidence for lateral gene transfer (LGT) of mrk alleles. All K. pneumoniae chromosomal alleles cluster in Clade A, along with several plasmid-borne or chromosomal alleles from $E$. coli. In some cases, the $K$. pneumoniae and $E$. coli alleles are identical (e.g. Ec_pOLA52/Kp_M20; EcM202/Kp_MGH78578). Similarly, in clade $\mathrm{B}$, two identical $E$. coli mrk $A B C D$ sequences (M184 and ECOR28) share high nucleotide sequence identity (98\%) to the plasmid-borne $K$. pneumoniae pIA565 mrkABCD. The mrkABCD concatenated nucleotide sequences of $K$. pneumoniae pIA565 (clade B) and K. pneumoniae MGH78578 (clade A) share only $78.2 \%$ nucleotide sequence identity. When analysed individually, the $m r k A, m r k B, m r k C$ and $m r k D$ gene fragment alignments produced essentially the same tree topology as the concatenated sequence (data not shown) with little variation in within-group diversity (Table 1 ). In contrast to other chaperone-usher systems, the $m r k D$ adhesin is more divergent than the mrkA major subunit and contributes the most of all $m r k$ alleles to the inter-group diversity (Table 1).

\section{Sequence comparison of the mrk locus from strains of $C$. freundii, C. koseri, E. coli and K. oxytoca}

We compared the $m r k$ gene clusters from representatives of each of the five clades: $5 \mathrm{mrk}$ regions were available from GenBank, 3 were sequenced in this study (Fig. 2). As expected, the mrkABCD gene order is conserved in all clades. Predicted insertion sequences were identified flanking both ends of the pMAS2027 and pOLA52 clusters (clade A), and at the $5^{\prime}$ end of clusters from ECOR28 (clade B) and C. freundii M46 (clade C), indicative of recent lateral gene transfer. Downstream of $m r k F$, a conserved 717 bp gene was present in five of the strains, including one from each of the five defined clades. This gene (labelled cko_00966 and kpn_03274 in the genomes of C. koseri ATCC BAA895 and $K$. pneumoniae MGH78578, respectively) encodes a central EAL domain (Pfam:PF00563, E = 1.7e-29) suggesting that it may have a role in signalling, however, no close homologs have been functionally characterised. PCR primers designed from these sequences demonstrated that this region was also 


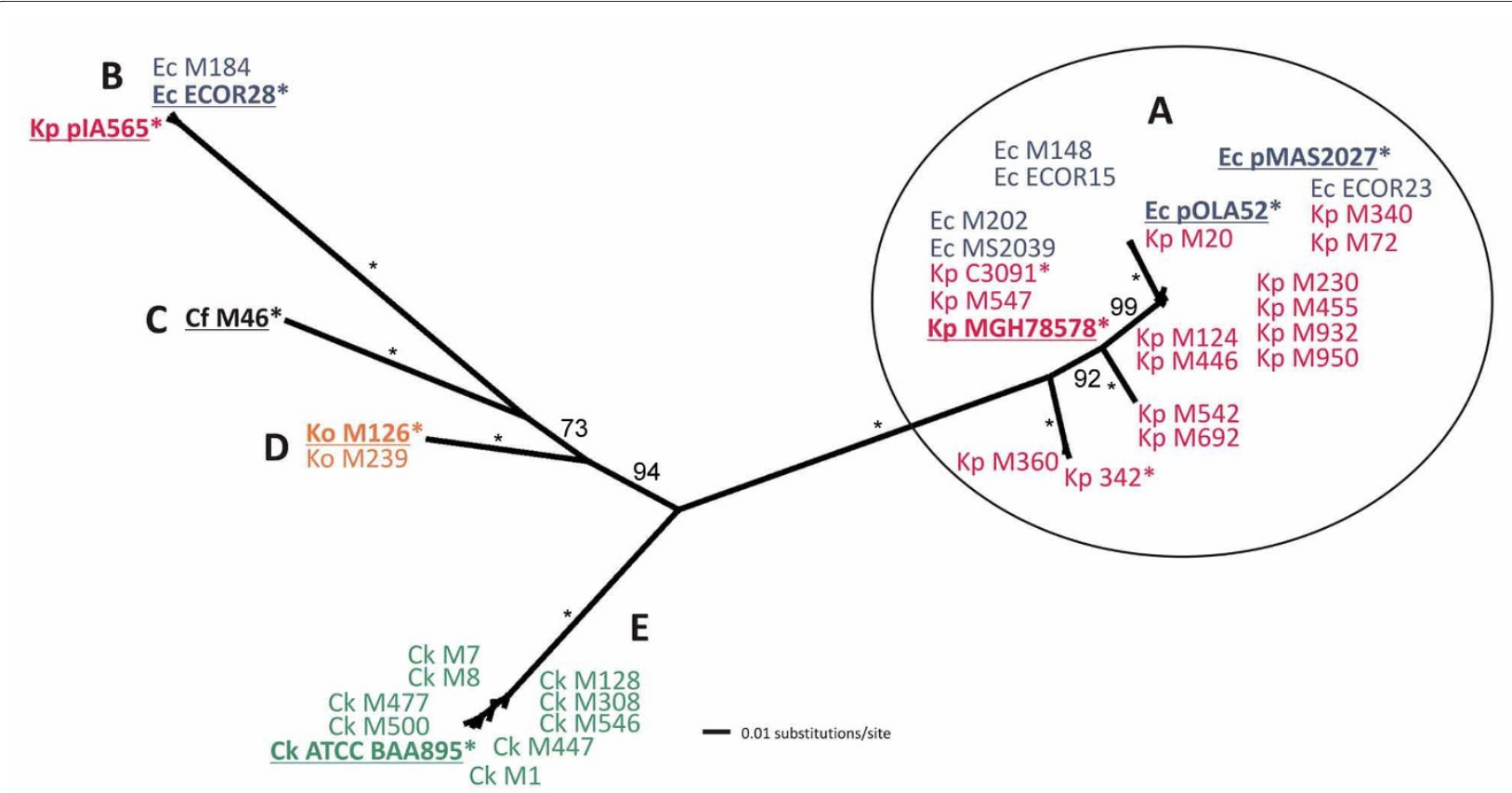

Figure 1 Unrooted consensus phylogram of the concatenated $m r k A B C D$ nucleotide fragments. Majority-rule consensus tree was based on 500 bootstrap replicates using dnaml, the DNA maximum likelihood algorithm implemented by PHYLIP [54]. Five well-supported clades are labelled A-E; the largest clade, A, is circled. Bootstrap values are shown; small asterisks next to branches denote $100 \%$ support. Taxon IDs include species name abbreviations as suffixes (Cf, C. freundii indicated in black; Ck, C. koseri indicated in green; Ec, E. coli indicated in blue; Ko, K. oxytoca indicated in orange; and Kp, K. pneumoniae indicated in red), followed by the strain name. Taxon IDs highlighted in bold and underlined refer to those used in further analyses of the complete sequence of their respective mrk locus. Complete mrk locus sequences available from GenBank are marked with a large asterisk next to the strain name.

conserved in 24 other strains examined (data not shown). Notably, cko_00966 homologs were not encoded downstream of the plasmid-borne mrk clusters in $E$. coli pMAS2027 and pOLA52, and there is no corresponding sequence information available for this region in pIA565 [37]. The putative $m r k E$ regulatory gene originally identified in pIA565 [37] was not present in any of the strains examined.
Type 3 fimbriae are functionally expressed in $C$. freundii, $C$. koseri, E. coli, K. oxytoca and K. pneumoniae

All of the $m r k$-positive strains examined in this study mediated mannose-resistant hemagglutination of tannic acid treated human RBC (MR/K agglutination), indicating they produced type 3 fimbriae. To specifically demonstrate a direct association between MR/K agglutination and type 3 fimbriae, the mrk locus was deleted from thirteen strains (E. coli MS2027, M184, ECOR15, ECOR28; K. pneumoniae M20, M124, M446, M542, M692; K. oxy-

Table 1: Diversity of individual $m r k A, m r k B, m r k C$ and $m r k D$ nucleotide sequences

\begin{tabular}{|c|c|c|c|c|c|c|c|c|}
\hline \multirow[b]{2}{*}{ Gene } & \multirow[b]{2}{*}{ Length } & \multicolumn{3}{|c|}{ Diversity Within Group (\%) } & \multicolumn{3}{|c|}{ Diversity Between Group (\%) } & \multirow[b]{2}{*}{$B$ and $E$} \\
\hline & & A & B & $\mathbf{E}$ & Mean & $A$ and $B$ & $A$ and $E$ & \\
\hline$m r k A$ & $403 \mathrm{nt}$ & 2.3 & 0.2 & 2.5 & 13.8 & 14.7 & 15.6 & 11.8 \\
\hline$m r k B$ & $246 \mathrm{nt}$ & 1.0 & 0.8 & 1.3 & 9.8 & 12.2 & 14.0 & 8.9 \\
\hline$m r k C$ & $655 \mathrm{nt}$ & 2.1 & 0.3 & 0.6 & 13.5 & 18.4 & 19.6 & 12.2 \\
\hline$m r k D$ & $506 \mathrm{nt}$ & 3.3 & 0.3 & 0.3 & 28.1 & 38.2 & 26.7 & 33.3 \\
\hline
\end{tabular}

1 Mean within group diversity; Group $C$ and Group D excluded as they contain a single sequence, and two identical sequences, respectively. 2Mean between group diversity calculated with all five groups. 


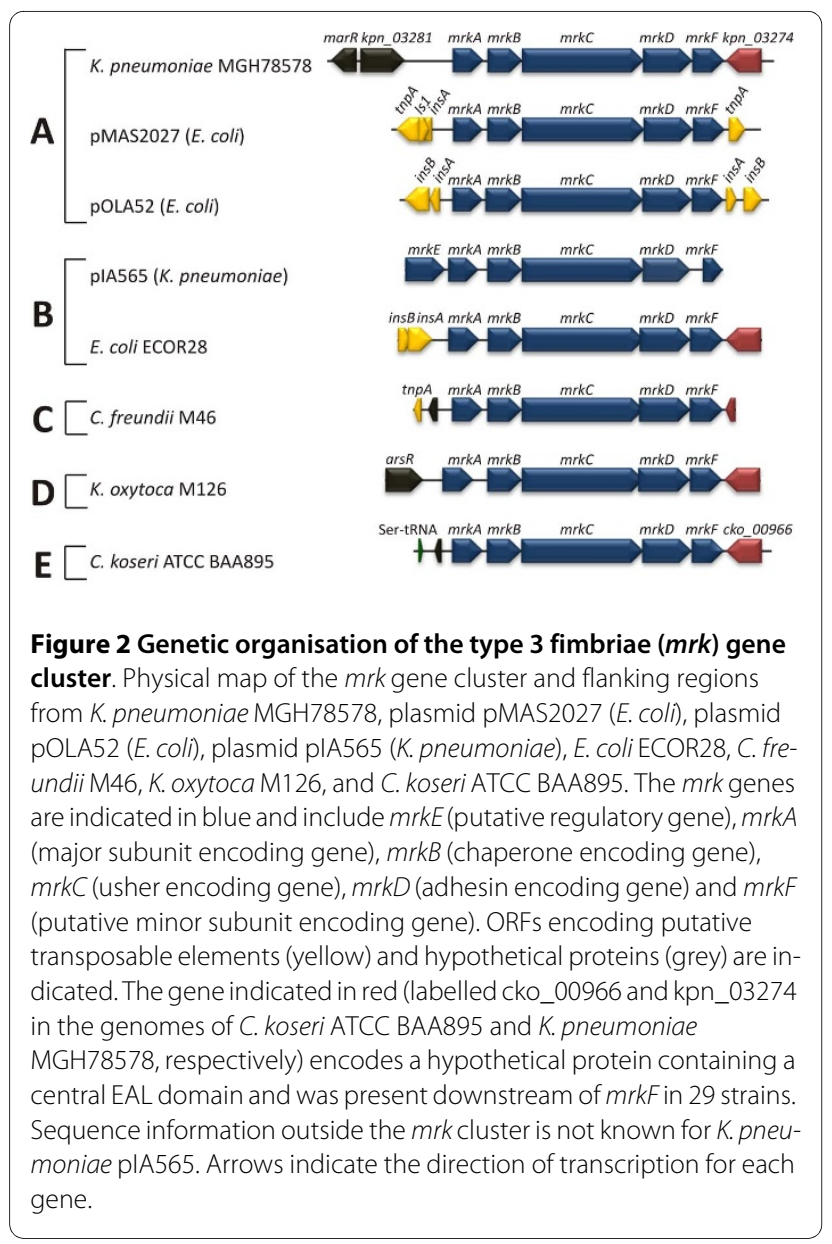

toca M126, M239; C. freundii M46; C. koseri M546) employing $\lambda$-red mediated homologous recombination. The strains were selected on the basis of their transformation efficiency and included at least one representative from each of the mrk phylogenetic clades. Several different assays were employed to compare the thirteen sets of wild-type and mrk deletion strains. First, SDS-PAGE analysis of crude cell lysates and subsequent Western blotting was performed using a type 3 fimbriae-specific antiserum. A predominant $15 \mathrm{kDa}$ band representing the MrkA major subunit was detected from all wild-type strains except $C$. freundii M46, which failed to react positively in this assay. In contrast, no reaction was observed for any of the mrk deletion mutants (Fig. 3 and data not shown). Next, the wild-type and mrk mutant strains were compared for their ability to mediate MR/K agglutination. Only the wild-type strains produced a positive phenotype (Fig. 4 and data not shown). Finally, the presence of type 3 fimbriae was confirmed by immunogold labelling employing type 3 fimbriae-specific antiserum for $E$. coli ECOR15 and C. koseri M546, but was absent in their corresponding mrk deletion mutants (Fig. 5). Taken together, the results demonstrate that MR/K agglutina- tion is a conserved phenotype for a range of Gram-negative organisms that express functional type 3 fimbriae.

\section{Type 3 fimbriae are strongly associated with biofilm formation}

The thirteen sets of isogenic wild-type and mrk deletion strains generated above were examined for their ability to produce a biofilm following growth in M9 minimal medium (containing $0.2 \%$ glucose) under dynamic culture conditions. Strong biofilm growth was observed from all wild-type strains except C. freundii M46. In contrast, deletion of the mrk gene cluster caused a significant reduction in biofilm growth $(p<0.0001)$ in all strains except E. coli M184 (Fig. 6). Similar results were also observed following growth in synthetic urine (data not shown). Thus, type 3 fimbriae contribute significantly to biofilm formation when expressed in E. coli, K. pneumoniae, $K$. oxytoca and C. koseri.

\section{Discussion}

Type 3 fimbriae are adhesive organelles produced by a range of Gram-negative pathogens that cause CAUTI. Here we show that type 3 fimbriae $(m r k A B C D)$ genes from 33 CAUTI isolates representing $C$. freundii, C. koseri, E. coli, K. oxytoca and K. pneumoniae cluster into five well-supported clades on the basis of nucleotide sequence. Type 3 fimbriae were expressed by all of these strains as indicated by their positive MR/K agglutination. Type 3 fimbrial expression was also associated with biofilm growth in the majority of these strains. This is the first report describing the distinct grouping of type 3 fimbrial genes into phylogenetic clades at the species level, with strong evidence supporting inter-species lateral gene transfer. We also demonstrate the functional expression of type 3 fimbriae by strains of C. koseri and C. freundii.

Phylogenetic analysis with individual and concatenated mrk $A B C D$ sequences revealed five distinct clades (A-E) which were strongly supported by long internal branches. The majority of the sequences grouped in clade $\mathrm{A}$, which is represented by the chromosomal mrk gene cluster from the genome sequenced $K$. pneumoniae strain MGH78578. Clades A and B contained $m r k$ gene clusters from K. pneumoniae (both chromosomal and plasmid origin) and E. coli (plasmid origin). Two mrk loci have been fully sequenced from $E$. coli; in both cases the $m r k$ genes are located on a conjugative plasmid (pMAS2027 and pOLA52, respectively) and flanked by transposonlike sequences $[30,40]$. While the genomic location of the $m r k$ genes in the additional seven E. coli strains identified in this study remains to be determined, the data presented here and in previous studies strongly suggests inter-genera lateral gene transfer of the mrk cluster $[17,28]$. In contrast, the composition of clade $\mathrm{E}$ is entirely 


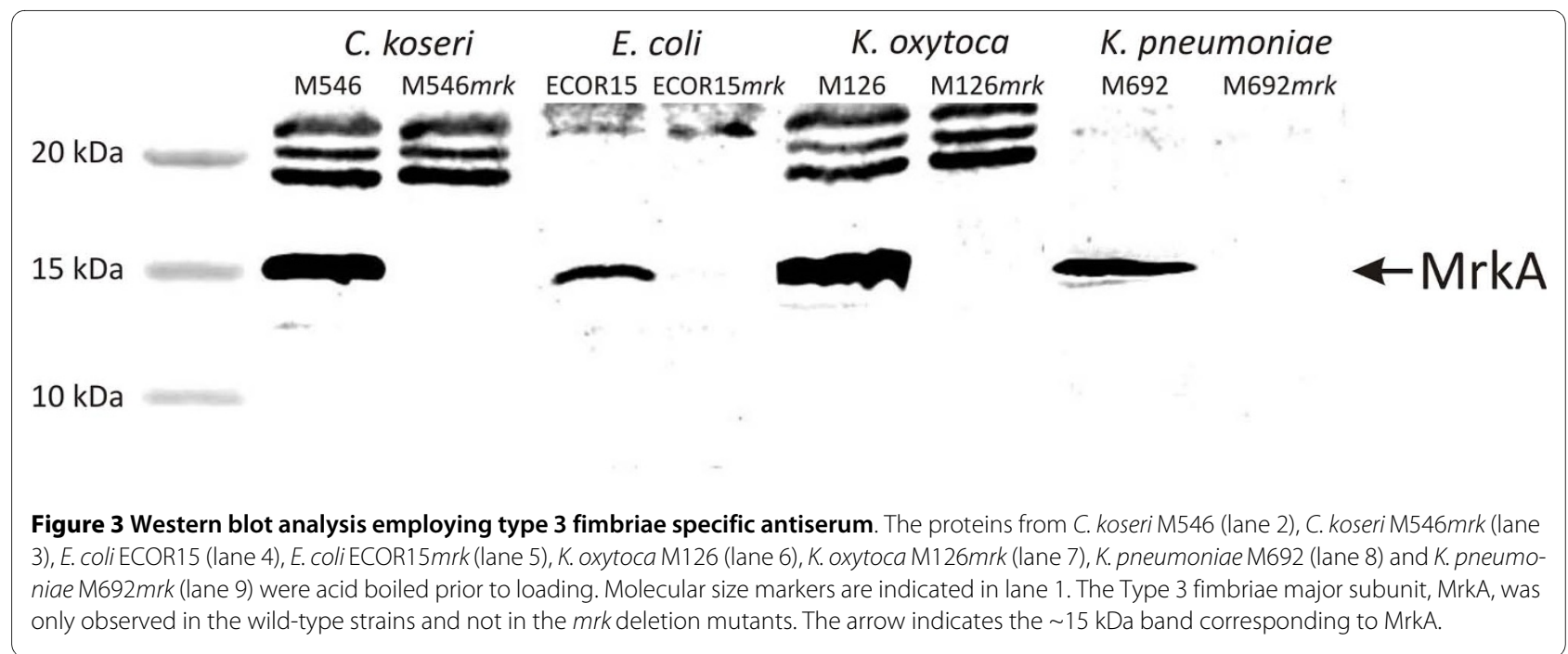

C. koseri sequences, while clades $\mathrm{C}$ and $\mathrm{D}$ are represented by a unique sequence from $C$ freundii and $K$. oxytoca, respectively. The presence of cko_00966 homologs downstream of representative $m r k$ clusters in all 5 clades strongly suggests that the ancestral $m r k A B C D$ locus was also encoded next to a cko_00966 homolog and that the clades are largely related by linear descent. Notably, the relationship determined here is not congruent with the known evolutionary relationship of Klebsiella, Citrobacter, and E. coli [41], supporting the occurrence of lateral gene transfer. We propose that clade A represents the $K$. pneumoniae lineage, with $m r k$ regions laterally transferred to E. coli (e.g. pMAS2027 and pOLA52) and clade $\mathrm{E}$ represents the $C$. koseri lineage. Clades B, C and D, which contain mrk sequences from $K$. pneumoniae, $E$. coli, C. freundii and K. oxytoca, are clearly under-represented and additional type 3 fimbrial gene sequences are required to confirm the groupings.
Among the four genes used in the phylogenetic analysis, $m r k D$ exhibited the highest inter-group diversity (Table 1). Thus, from the partial sequence comparisons performed in this work, the MrkD adhesin displayed greater sequence variability than the MrkA major subunit. This is inconsistent with other chaperone-usher fimbriae such as type 1 and $\mathrm{P}$ fimbriae, where the sequence of the adhesin (e.g. FimH, PapG) is more conserved than the major subunit protein (e.g. FimA, PapA). We note, however, that these findings require substantiation via comparison of the entire sequence of each structural subunit from multiple strains. The MrkD adhesin mediates several phenotypes, including MR/K agglutination, as well as adherence to human endothelial cells, urinary bladder cells, basement membranes and ECM proteins such as collagen IV and V $[5,31,34,35]$. Interestingly, previous studies have demonstrated that sequence variations in the MrkD adhesin are associated with differential binding properties [42-44]. Our study demon-

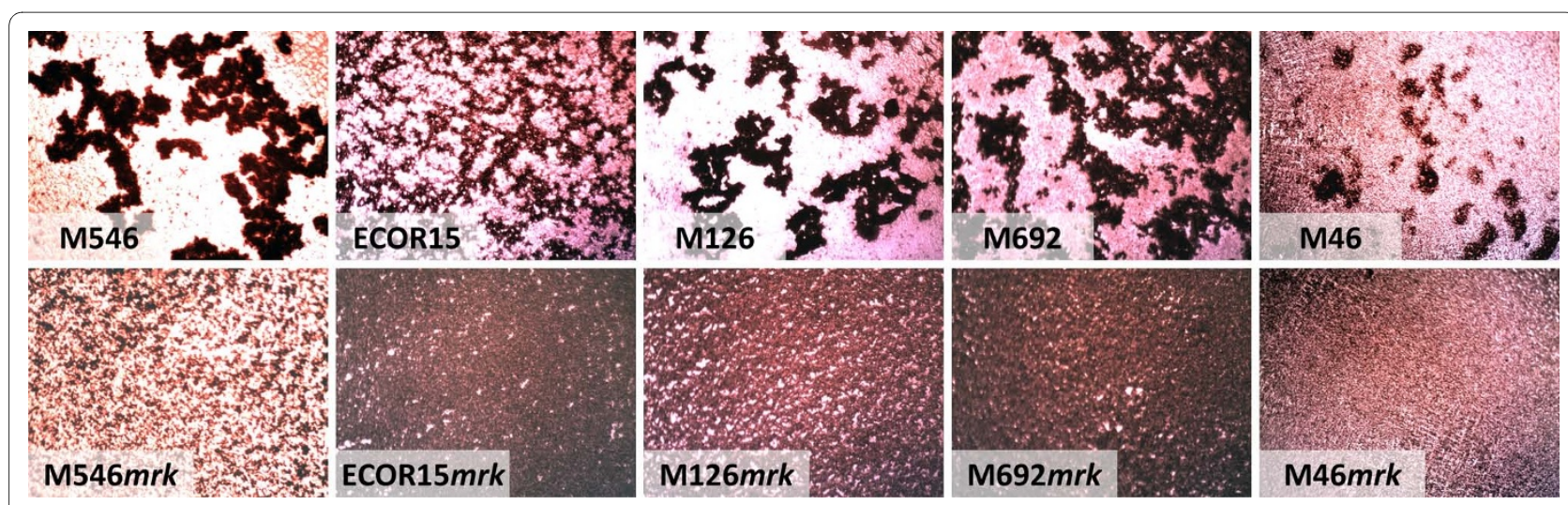

Figure 4 Phase contrast microscopy illustrating MR/K agglutination. Parental wild-type strains C. koseri M546, E. coli ECOR15, K. oxytoca M126, K. pneumoniae M692 and C. freundii M46 demonstrated strong agglutination of tannic acid treated human erythrocytes, while their corresponding mrk deletion mutants, M546mrk, ECOR15mrk, M126mrk, M692mrk and M46mrk were negative for agglutination. 


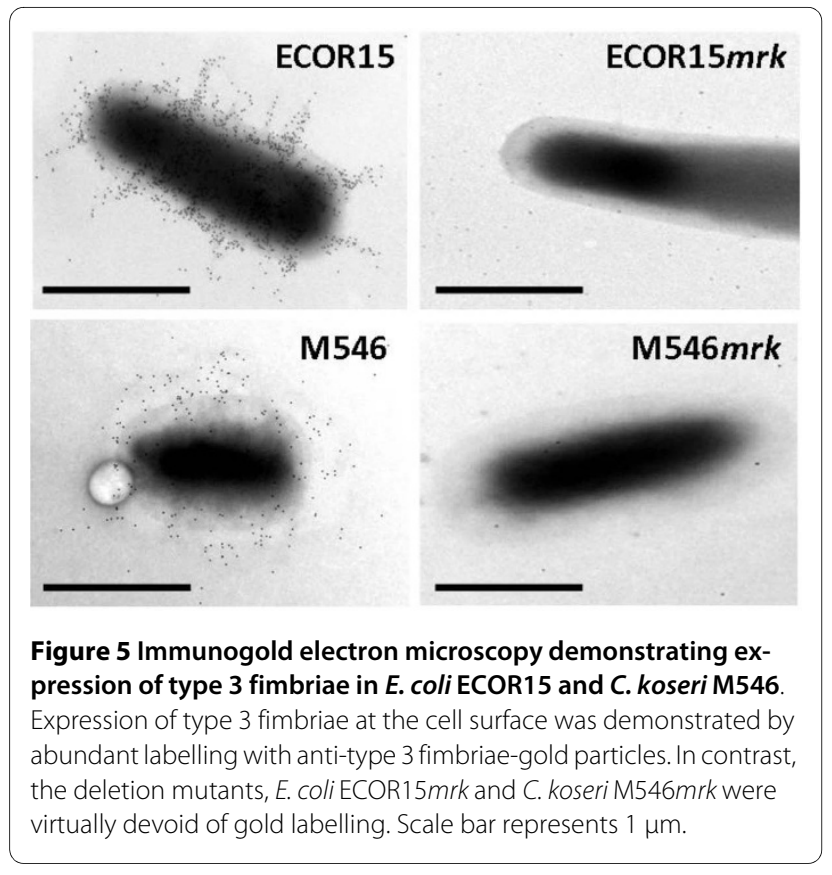

strates that the degree of sequence variation in MrkD might be even greater than previously predicted [44].

CAUTI is associated with biofilm formation on the inner surface of indwelling catheters. Thirteen independent mrk deletion mutants were generated and used to examine type 3 fimbriae associated phenotypes including $\mathrm{MR} / \mathrm{K}$ agglutination and biofilm formation. All of the $m r k$ mutants were unable to cause MR/K agglutination, confirming that this property is highly specific for type $3 \mathrm{fim}$ briae. In biofilm assays, 11/13 mrk mutants displayed a significant reduction in biofilm growth compared to their respective parent strain, demonstrating that type 3 fimbriae contribute to this phenotype across a range of different genera and species. The exceptions were $C$. freundii M46 and E. coli M184. C. freundii M46 failed to produce a significant biofilm in the assay conditions employed irrespective of its mrk genotype. Although this strain caused MR/K agglutination, we were also unable to detect the MrkA major subunit protein by western blot analysis. E. coli M184 showed no reduction in biofilm growth upon deletion of the $m r k$ genes. It is likely that $E$. coli M184 contains additional mechanisms that promote biofilm growth and therefore deletion of the mrk genes did not result in loss of this phenotype.

\section{Conclusions}

This study demonstrated that the expression of functional type 3 fimbriae is common to many Gram-negative pathogens that cause CAUTI. Biofilm growth mediated by type 3 fimbriae may be important for the survival of these organisms on the surface of urinary catheters and within the hospital environment. Although our analysis provides additional evidence for the spread of type 3 fimbrial genes by lateral gene transfer, further work is required to substantiate the clade structure reported here by examining more strains as well as other genera that make type 3 fimbriae and cause CAUTI such as Proteus and Providentia.

\section{Methods}

\section{Bacterial strains, plasmids \& growth conditions}

The strains and plasmids used in this study are described in Table 2. Clinical UTI isolates were obtained from urine samples of patients at the Princess Alexandra Hospital (Brisbane, Australia) and have been described previously [45]. E. coli ECOR15, ECOR23 and ECOR28 were from the E. coli reference (ECOR) collection [46]. Cells were routinely grown at $37{ }^{\circ} \mathrm{C}$ on solid or in liquid Luria-Bertani (LB) medium supplemented with appropriate antibiotics unless otherwise stated. M9 minimal medium and synthetic urine were formulated as previously described $[47,48]$.

\section{DNA manipulations and genetic techniques}

Plasmid DNA was isolated using the QIAprep Spin Miniprep Kit (Qiagen, Australia). Restriction endonucleases were used according to the manufacturer's specifications (New England Biolabs, USA). Chromosomal DNA was purified as previously described [48]. PCR was performed using Taq polymerase according to the manufacturer's instructions (New England Biolabs, USA). DNA sequencing was performed by the Australian Equine Genome Research Centre. Deletion mutants were constructed essentially as previously described using either pKD46 [49] or pKOBEG199 [50,51], with the exception that $C$. freundii and C. koseri strains were heated at $42^{\circ} \mathrm{C}$ for 2 min prior to electroporation. Primers used to generate deletion mutants were as follows: 1293 and 1294 (E. coli MS2027), 1456 and 1457 (E. coli ECOR15 and K. pneumoniae strains), 1458 and 1459 (E. coli ECOR28), 1456 and 1459 (E. coli M184), 1460 and 1459 (K. oxytoca strains), 1456 and 1461 (C. koseri M546), 1462 and 1459 (C. freundii M46) (Table 3). All deletion mutants were checked by PCR using specific primers (Table 3 ) in conjunction with primers targeting the kanamycin or chloramphenicol resistance gene [49] and further confirmed by sequencing. Sequence information outside the mrk cluster was obtained by inverse PCR (using primer combinations 1450/1452, 1450/1454, 1450/1453, 1451/ 1455 , or $1451 / 1453$ ) or standard PCR employing primers designed from the genome sequenced $K$. pneumoniae MGH78578 or C. koseri ATCC BAA895 (Table 3).

\section{MR/K agglutination}

Bacterial agglutination of tannic acid treated human erythrocytes (MR/K agglutination) was performed as 


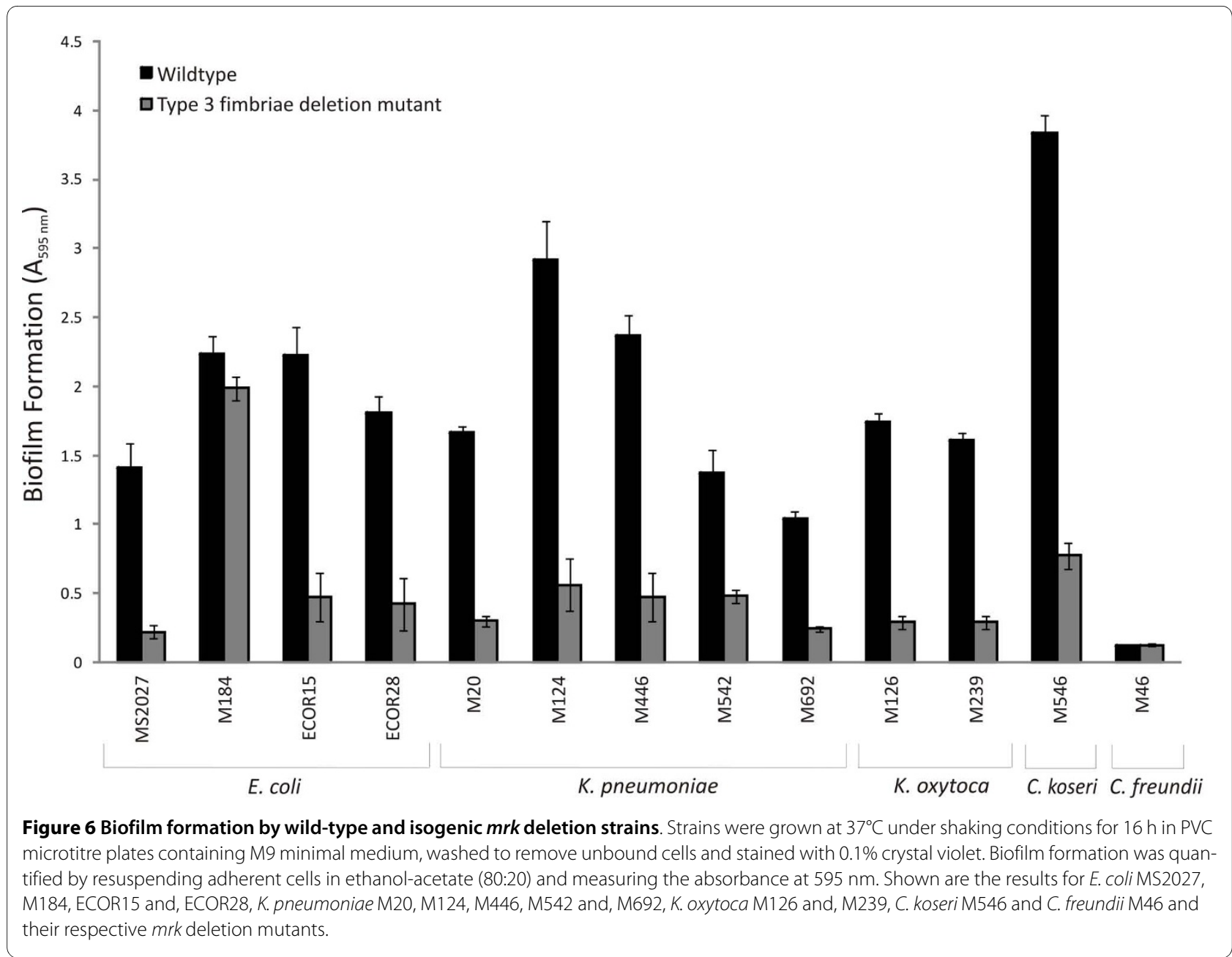

previously described to detect the expression of Type 3 fimbriae [29]. Bacterial strains were grown overnight as shaking cultures in M9 minimal medium. Strains which produced a negative result in this assay were enriched for type 3 fimbriae production by three successive rounds of $48 \mathrm{~h}$ static growth in M9 minimal medium and then retested.

\section{Biofilm study}

Biofilm formation on polyvinyl chloride (PVC) surfaces was monitored by using 96-well microtitre plates (Falcon) essentially as previously described [16]. Briefly, cells were grown for $24 \mathrm{~h}$ in $\mathrm{M} 9$ minimal medium (containing $0.2 \%$ glucose) or $48 \mathrm{~h}$ in synthetic urine at $37^{\circ} \mathrm{C}$ under shaking conditions, washed to remove unbound cells and stained with crystal violet. Quantification of biofilm mass was performed by addition of acetone-ethanol (20:80) and measurement of the dissolved crystal violet at an optical density of $595 \mathrm{~nm}$. All experiments were performed in a minimum of eight replicates.

\section{Immunoblotting and immunogold-labelled electron microscopy}

Crude cell lysates were prepared from overnight cultures and boiled in acid as previously described [14]. Protein samples were analysed by SDS-PAGE and western blotting as previously described [52] employing a type 3 fimbriae specific antiserum. Immunogold labelling was performed using the same Type 3 fimbriae specific antiserum as previously described [14]. Cells were examined under a JEOL JEM1010 TEM operated at $80 \mathrm{kV}$. Images were captured using an analysis Megaview digital camera.

\section{Phylogenetic and sequence analysis}

PCR products were generated from an internal region of $m r k A$ (416 bp), $m r k B$ (243 bp), $m r k C$ (657 bp) and $m r k D$ (778), respectively, from each of the 33 CAUTI strains and sequenced on both strands. These sequences correspond to nucleotides 112 to 530 of $m r k A, 66$ to 308 of $m r k B, 173$ to 829 of $m r k C$ and 157 to 934 of $m r k D$ in the reference strain K. pneumoniae MGH78578 (CP000647). Individual and concatenated gene fragments from the 33 
Table 2: Bacterial strains and plasmids used in this study

\begin{tabular}{|c|c|c|}
\hline Strains/Plasmids & Description & Reference \\
\hline \multicolumn{3}{|l|}{ Strains } \\
\hline MS2027 & E. coli CAUTI isolate & [28] \\
\hline M20 & K. pneumoniae CAUTI isolate & [28] \\
\hline M46 & C. freundii $\mathrm{ABU}$ isolate & [28] \\
\hline M124 & K. pneumoniae CAUTI isolate & [28] \\
\hline M126 & K. oxytoca CAUTI isolate & [28] \\
\hline M184 & E. coli pyelonephritis isolate & [28] \\
\hline M239 & K. oxytoca CAUTI isolate & [28] \\
\hline M446 & K. pneumoniae CAUTI isolate & [28] \\
\hline M542 & K. pneumoniae CAUTI isolate & [28] \\
\hline M546 & C. koseri CAUTI isolate & [28] \\
\hline M692 & K. pneumoniae CAUTI isolate & [28] \\
\hline MS2181 & CAUTI E. coli MS2027mrk:: cam & This study \\
\hline MS2266 & Pyelonephritis E. coli M184mrk::cam & This study \\
\hline MS2267 & E. coli ECOR15mrk::cam & This study \\
\hline MS2332 & CAUTI K. pneumoniae M124mrk::kan & This study \\
\hline MS2334 & CAUTI K. pneumoniae M446mrk::kan & This study \\
\hline MS2335 & CAUTI K. pneumoniae M542mrk::kan & This study \\
\hline MS2374 & CAUTI K. pneumoniae M20Ark::kan & This study \\
\hline MS2377 & CAUTI K. oxytoca M126mrk::kan & This study \\
\hline MS2379 & CAUTI K. oxytoca M239mrk:: kan & This study \\
\hline MS2454 & CAUTI C. koseri M546mrk::kan & This study \\
\hline MS2456 & ABU C. freundii M46mrk::kan & This study \\
\hline MS2458 & E. coli ECOR28mrk::kan & This study \\
\hline MS2515 & CAUTI K. pneumoniae M692mrk::kan & This study \\
\hline \multicolumn{3}{|l|}{ Plasmids } \\
\hline pKD3 & Deletion mutant template plasmid (cam) & [49] \\
\hline pKD4 & Deletion mutant template plasmid (kan) & [49] \\
\hline pKD46 & Temperature-sensitive plasmid containing $\lambda$-Red recombinase system & [49] \\
\hline pKOBEG199 & Plasmid with $\lambda$-Red genes under the control of the arabinose-inducible promoter & [50] \\
\hline
\end{tabular}

CAUTI strains (and six additional mrk sequences available at GenBank from strains causing other infections; accession numbers: CP000647, EU682505, CP000964, M55912, CP000822, EU370913) were aligned using ClustalX [53], and subjected to phylogenetic analysis using PHYLIP [54]. Maximum likelihood (ML) trees were built from a concatenated alignment of 2104 nucleotides (comprising 1269 conserved sites and 775 informative sites) using the dnaml algorithm in PHYLIP [54]. A consensus tree of $500 \mathrm{ML}$ bootstrap replicates was prepared using the majority rule method as implemented by Split- stree version $4[55,56]$. We were unable to amplify $m r k D$ from $E$. coli M202 and only used the $m r k A B C$ concatenated fragments in the analysis. For comparative analysis, the complete $m r k$ cluster (and adjacent regions) from $E$. coli ECOR28, C. freundii M46 and K. oxytoca M126 were amplified using an inverse PCR strategy and sequenced.

\section{Statistical analysis}

Differences in biofilm formation between wild-type and mrk mutant strains were analysed using the ANOVA single factor test (Minitab 15 Statistical Software). 


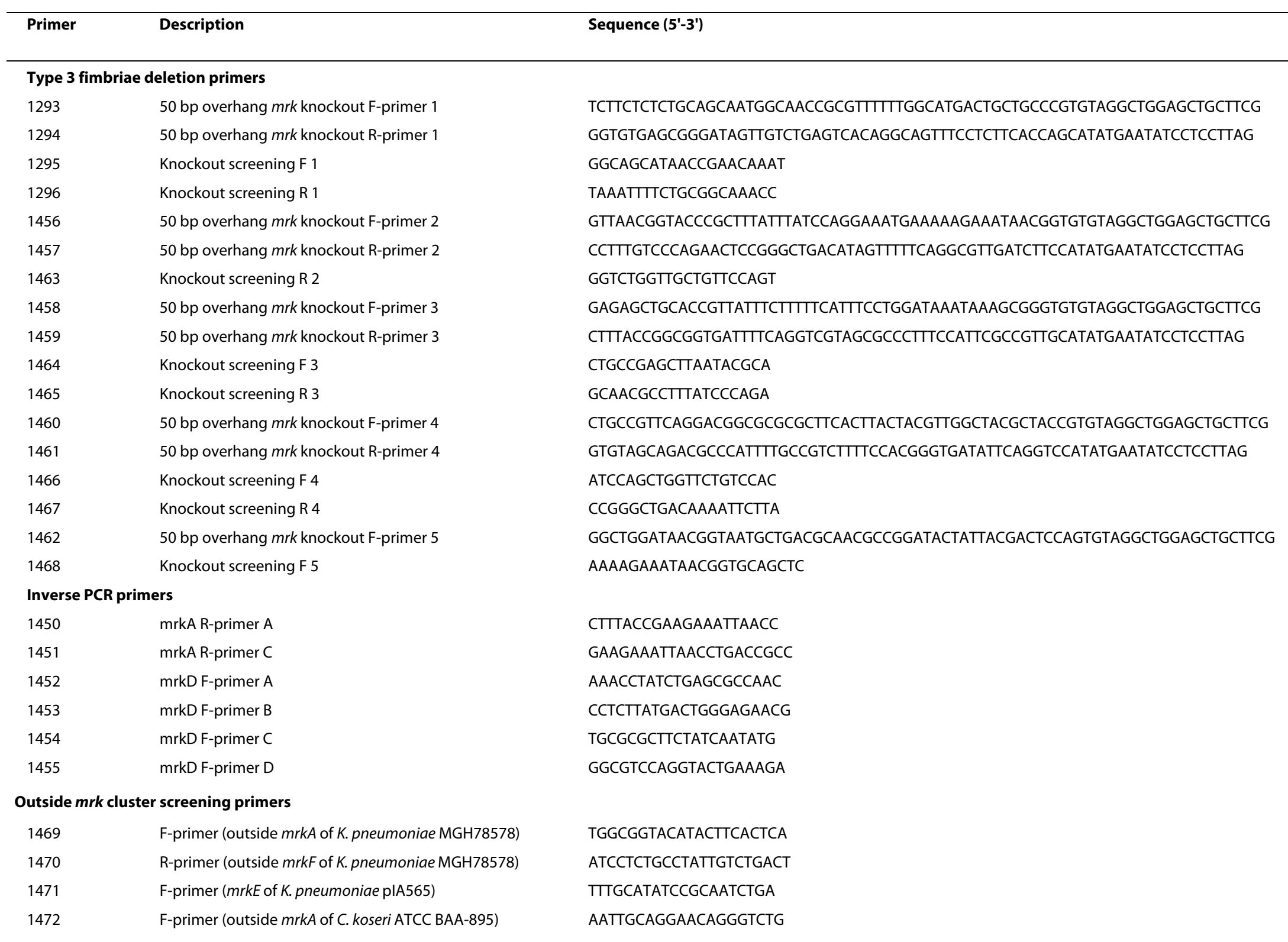


Table 3: Primers used in this study (Continued)

$\begin{array}{lll}1473 & \text { R-primer (outside } m r k F \text { of } \text { C. koseri ATCC BAA-895) } & \text { TTCCTTCCCGTAACCA } \\ 1474 & \text { F-primer (outside } m r k \text { of } E \text {. coli MS2027) } & \text { AGCGTCTGCATGGCTTAT } \\ 1475 & \text { R-primer (outside } m r k F \text { of } E \text {. coli MS2027) } & \text { AACGACGTTGGGTCGTTAGT } \\ \text { pMAS2027 screening primers } & \\ 1476 & \text { pMAS2027 screening primer F1 } & \text { GCGCAGAACTGGTTATGAAT } \\ 1477 & \text { pMAS2027 screening primer R1 } & \text { TCATGGATTTCTTCCTAACAA } \\ 1478 & \text { pMAS2027 screening primer F2 } & \text { ACAACTATCCCGCTCACACC } \\ 1479 & \text { pMAS2027 screening primer R2 } & \text { ACCGTAACGCGTAGTCACC } \\ 1480 & \text { pMAS2027 screening primer F3 } & \text { TGCTTCAGCAGCATATCAGG } \\ 1481 & \text { pMAS2027 screening primer R3 } & \text { GGAAAGCGTAAAGCAGGTG } \\ 1482 & \text { pMAS2027 screening primer F4 } & \text { CCGTATGCGCTTITCAAGT } \\ 1483 & \text { pMAS2027 screening primer R4 } & \text { AAAGTGAAGCCCGCTTCT } \\ 1484 & \text { pMAS2027 screening primer F5 } & \text { ACGGGTAAGACCGCTAACCT } \\ 1485 & \text { pMAS2027 screening primer R5 } & \text { TCGATAAGGTAGGCATCAACAA } \\ \end{array}$




\section{Nucleotide sequence accession numbers}

Gene fragments were deposited in GenBank under the accession numbers: FJ96754, FJ96756-FJ96774, and FJ96777-FJ96789 (for $m r k A$ ), FJ96793, FJ96795-FJ96811, FJ96813-FJ96814, and FJ96817-FJ96829 (for $m r k C$ ) and FJ96832, FJ96834-FJ96849, FJ96851-FJ96852, and FJ96855-FJ96867 (for $m r k D$ ). The $m r k B$ sequences were described previously [28]. The complete $m r k$ cluster (and adjacent regions) from E. coli ECOR28, C. freundii M46 and $K$. oxytoca M126 were deposited in GenBank under accession numbers FJ96870, FJ96871 and FJ96872, respectively.

\section{Ethical approval}

Approval for this study was obtained from the Princess Alexandra Hospital Human Research Ethics Committee (2005/098). Since the study used E. coli isolates collected as part of routine methods for the diagnosis of UTI and no additional procedures on patients were involved, individual informed consent was not obtained.

\section{Authors' contributions}

CYO carried out the majority of the experimental work under the supervision of AGM and MAS. SAB performed the bioinformatic analysis and contributed to writing the manuscript. MT performed the immunogold labelled electron microscopy and contributed to writing the manuscript. CF contributed to the construction of mutants and writing of the manuscript. AGM contributed to the design of experiments and writing of the manuscript. MAS conceived the study and wrote the manuscript. All authors read and approved the final manuscript.

\section{Acknowledgements}

This work was supported by grants from the National Health and Medical Research Council (455914 and 631654) and the Australian Research Council (DP0666852). SAB is supported by an ARC Australian Research Fellowship (DP0881247). We thank Prof Timo Korhonen for providing Type 3 fimbriae antiserum.

\section{Author Details}

${ }^{1}$ Centre for Infectious Disease Research, School of Chemistry and Molecular Biosciences, University of Queensland, Brisbane, Queensland 4072, Australia and 2Université de Clermont 1, UFR Pharmacie, Laboratoire de Bactériologie, 28 place Henri Dunant, Clermont-Ferrand, F-63001 France

Received: 5 February 2010 Accepted: 24 June 2010

Published: 24 June 2010

\section{References}

1. Stamm WE: Catheter-associated urinary tract infections: epidemiology, pathogenesis, and prevention. Am J Med 1991, 91(3B):65S-71S.

2. Warren JW, Tenney JH, Hoopes JM, Muncie HL, Anthony WC: A prospective microbiologic study of bacteriuria in patients with chronic indwelling urethral catheters. J Infect Dis 1982, 146(6):719-723.

3. Paterson DL, Lipman J: Returning to the pre-antibiotic era in the critically ill: the XDR problem. Crit Care Med 2007, 35(7):1789-1791.

4. Warren JW: Catheter-associated urinary tract infections. Int J Antimicrob Agents 2001, 17(4):299-303.

5. Sebghati TA, Korhonen TK, Hornick DB, Clegg S: Characterization of the type 3 fimbrial adhesins of Klebsiella strains. Infect Immun 1998, 66(6):2887-2894.

6. Giltner CL, van Schaik EJ, Audette GF, Kao D, Hodges RS, Hassett DJ, Irvin RT: The Pseudomonas aeruginosa type IV pilin receptor binding domain functions as an adhesin for both biotic and abiotic surfaces. $\mathrm{Mol}$ Microbiol 2006, 59(4):1083-1096.
7. Zogaj X, Bokranz W, Nimtz M, Romling U: Production of cellulose and curli fimbriae by members of the family Enterobacteriaceae isolated from the human gastrointestinal tract. Infect Immun 2003, 71(7):4151-4158.

8. Ghigo JM: Natural conjugative plasmids induce bacterial biofilm development. Nature 2001, 412(6845):442-445.

9. Reisner A, Haagensen JA, Schembri MA, Zechner EL, Molin S: Development and maturation of Escherichia coli K-12 biofilms. Mol Microbiol 2003, 48(4):933-946.

10. Valle J, Mabbett AN, Ulett GC, Toledo-Arana A, Wecker K, Totsika M, Schembri MA, Ghigo JM, Beloin C: UpaG, a new member of the trimeric autotransporter family of adhesins in uropathogenic Escherichia coli. J Bacteriol 2008, 190(12):4147-4161.

11. Kjaergaard K, Schembri MA, Ramos C, Molin S, Klemm P: Antigen 43 facilitates formation of multispecies biofilms. Environ Microbio/ 2000, 2(6):695-702.

12. Lane MC, Lockatell V, Monterosso G, Lamphier D, Weinert J, Hebel JR, Johnson DE, Mobley HL: Role of motility in the colonization of uropathogenic Escherichia coli in the urinary tract. Infect Immun 2005 , 73(11):7644-7656

13. Allsopp LP, Totsika M, Tree JJ, Ulett GC, Mabbett AN, Wells TJ, Kobe B, Beatson SA, Schembri MA: UpaH is a newly identified autotransporter protein that contributes to biofilm formation and bladder colonization by uropathogenic Escherichia coli CFT073. Infect Immun 2010, 78(4):1659-1669.

14. Ulett GC, Mabbett AN, Fung KC, Webb Rl, Schembri MA: The role of F9 fimbriae of uropathogenic Escherichia coli in biofilm formation. Microbiology 2007, 153(Pt 7):2321-2331.

15. Connell I, Agace W, Klemm P, Schembri M, Marild S, Svanborg C: Type 1 fimbrial expression enhances Escherichia coli virulence for the urinary tract. Proc Natl Acad Sci USA 1996, 93(18):9827-9832.

16. Schembri MA, Klemm P: Biofilm formation in a hydrodynamic environment by novel fim $\mathrm{H}$ variants and ramifications for virulence. Infect Immun 2001, 69(3):1322-1328.

17. Burmolle M, Bahl MI, Jensen LB, Sorensen SJ, Hansen LH: Type 3 fimbriae, encoded by the conjugative plasmid pOLA52, enhance biofilm formation and transfer frequencies in Enterobacteriaceae strains. Microbiology 2008, 154(Pt 1):187-195.

18. Hornick DB, Allen BL, Horn MA, Clegg S: Fimbrial types among respiratory isolates belonging to the family Enterobacteriaceae. J Clin Microbiol 1991, 29(9):1795-1800.

19. Yakubu DE, Old DC, Senior BW: The haemagglutinins and fimbriae of Proteus penneri. J Med Microbiol 1989, 30(4):279-284.

20. Old DC, Adegbola RA: Antigenic relationships among type-3 fimbriae of Enterobacteriaceae revealed by immunoelectronmicroscopy. J Med Microbiol 1985, 20(1):113-121.

21. Adegbola RA, Old DC: Fimbrial and non-fimbrial haemagglutinins in Enterobacter aerogenes. J Med Microbiol 1985, 19(1):35-43.

22. Old DC, Adegbola RA: Relationships among broad-spectrum and narrow-spectrum mannose-resistant fimbrial hemagglutinins in different Yersinia species. Microbiol Immunol 1984, 28(12):1303-1311.

23. Adegbola RA, Old DC, Senior BW: The adhesins and fimbriae of Proteus mirabilis strains associated with high and low affinity for the urinary tract. J Med Microbiol 1983, 16(4):427-431.

24. Adegbola RA, Old DC, Aleksic S: Rare MR/K-like hemagglutinins (and type-3-like fimbriae) of Salmonella strains. FEMS Microbiol Lett 1983, 19(2-3):233-238

25. Adegbola RA, Old DC: Fimbrial haemagglutinins in Enterobacter species. $J$ Gen Microbiol 1983, 129(7):2175-2180.

26. Old DC, Adegbola R, Scott SS: Multiple fimbrial haemagglutinins in Serratia species. Med Microbiol Immunol 1983, 172(2):107-115.

27. Old DC, Adegbola RA: Haemagglutinins and fimbriae of Morganella, Proteus and Providencia. J Med Microbiol 1982, 15(4):551-564.

28. Ong CL, Ulett GC, Mabbett AN, Beatson SA, Webb RI, Monaghan W, Nimmo GR, Looke DF, McEwan AG, Schembri MA: Identification of type 3 fimbriae in uropathogenic Escherichia coli reveals a role in biofilm formation. J Bacterio/ 2008, 190(3):1054-1063.

29. Duguid JP: Fimbriae and adhesive properties in Klebsiella strains. J Gen Microbiol 1959, 21:271-286.

30. Ong CL, Beatson SA, McEwan AG, Schembri MA: Conjugative plasmid transfer and adhesion dynamics in an Escherichia coli biofilm. Appl Environ Microbiol 2009, 75(21):6783-6791. 
31. Jagnow J, Clegg S: Klebsiella pneumoniae MrkD-mediated biofilm formation on extracellular matrix- and collagen-coated surfaces. Microbiology 2003, 149(Pt 9):2397-2405.

32. Boddicker JD, Anderson RA, Jagnow J, Clegg S: Signature-tagged mutagenesis of Klebsiella pneumoniae to identify genes that influence biofilm formation on extracellular matrix material. Infect Immun 2006 74(8):4590-4597.

33. Langstraat J, Bohse M, Clegg S: Type 3 fimbrial shaft (MrkA) of Klebsiella pneumoniae, but not the fimbrial adhesin (MrkD), facilitates biofilm formation. Infect Immun 2001, 69(9):5805-5812.

34. Sebghati TA, Clegg S: Construction and characterization of mutations within the Klebsiella mrkD1P gene that affect binding to collagen type V. Infect Immun 1999, 67(4):1672-1676.

35. Tarkkanen AM, Virkola R, Clegg S, Korhonen TK: Binding of the type 3 fimbriae of Klebsiella pneumoniae to human endothelial and urinary bladder cells. Infect Immun 1997, 65(4):1546-1549.

36. Tarkkanen AM, Allen BL, Westerlund B, Holthofer H, Kuusela P, Risteli L, Clegg S, Korhonen TK: Type V collagen as the target for type-3 fimbriae, enterobacterial adherence organelles. Mol Microbio/ 1990, 4(8):1353-1361.

37. Allen BL, Gerlach GF, Clegg S: Nucleotide sequence and functions of mrk determinants necessary for expression of type 3 fimbriae in Klebsiella pneumoniae. J Bacteriol 1991, 173(2):916-920.

38. Huang YJ, Liao HW, Wu CC, Peng HL: MrkF is a component of type 3 fimbriae in Klebsiella pneumoniae. Res Microbiol 2009, 160(1):71-79.

39. Struve C, Bojer M, Krogfelt KA: Identification of a conserved chromosomal region encoding Klebsiella pneumoniae type 1 and type 3 fimbriae and assessment of the role of fimbriae in pathogenicity. Infect Immun 2009, 77(11):5016-5024.

40. Norman A, Hansen LH, She Q, Sorensen SJ: Nucleotide sequence of pOLA52: a conjugative IncX1 plasmid from Escherichia coli which enables biofilm formation and multidrug efflux. Plasmid 2008, 60(1):59-74.

41. Wertz JE, Goldstone C, Gordon DM, Riley MA: A molecular phylogeny of enteric bacteria and implications for a bacterial species concept. J Evol Biol 2003, 16(6):1236-1248

42. Hornick DB, Thommandru J, Smits W, Clegg S: Adherence properties of an mrkD-negative mutant of Klebsiella pneumoniae. Infect Immun 1995, 63(5):2026-2032.

43. Schurtz TA, Hornick DB, Korhonen TK, Clegg S: The type 3 fimbrial adhesin gene $(m r k D)$ of Klebsiella species is not conserved among all fimbriate strains. Infect Immun 1994, 62(10):4186-4191.

44. Huang YJ, Wu CC, Chen MC, Fung CP, Peng HL: Characterization of the type 3 fimbriae with different MrkD adhesins: possible role of the MrkD containing an RGD motif. Biochem Biophys Res Commun 2006, 350(3):537-542.

45. Mabbett AN, Ulett GC, Watts RE, Tree JJ, Totsika M, Ong CL, Wood JM, Monaghan W, Looke DF, Nimmo GR, et al:: Virulence properties of asymptomatic bacteriuria Escherichia coli. Int J Med Microbiol 2009 299(1):53-63.

46. Ochman H, Selander RK: Standard reference strains of Escherichia coli from natural populations. J Bacteriol 1984, 157(2):690-693.

47. Martino PD, Fursy R, Bret L, Sundararaju B, Phillips RS: Indole can act as an extracellular signal to regulate biofilm formation of Escherichia coli and other indole producing bacteria. Can J Microbio/ 2003, 49(7):443-449.

48. Sambrook J, Russell DW: Molecular Cloning: A Laboratory Manual. Volume 1. Third edition. New York: Cold Spring Harbor Laboratory Press; 2001.

49. Datsenko KA, Wanner BL: One-step inactivation of chromosomal genes in Escherichia coli K-12 using PCR products. Proc Natl Acad Sci USA 2000, 97(12):6640-6645

50. Balestrino D, Haagensen JA, Rich C, Forestier C: Characterization of type 2 quorum sensing in Klebsiella pneumoniae and relationship with biofilm formation. J Bacteriol 2005, 187(8):2870-2880.

51. Coudeyras S, Nakusi L, Charbonnel N, Forestier C: A tripartite efflux pump involved in gastrointestinal colonization by Klebsiella pneumoniae confers a tolerance response to inorganic acid. Infect Immun 2008, 76(10):4633-4641.

52. Ulett GC, Webb RI, Schembri MA: Antigen-43-mediated autoaggregation impairs motility in Escherichia coli. Microbiology 2006, 152(Pt 7):2101-2110.
53. Jeanmougin F, Thompson JD, Gouy M, Higgins DG, Gibson TJ: Multiple sequence alignment with Clustal X. Trends Biochem Sci 1998, 23(10):403-405

54. Felsenstein J: PHYLIP (Phylogeny Inference Package) version 3.6. Seattle: Department of Genome Sciences; 2004.

55. Kloepper TH, Huson DH: Drawing explicit phylogenetic networks and their integration into SplitsTree. BMC Evol Biol 2008, 8:22.

56. Huson DH: SplitsTree: analyzing and visualizing evolutionary data. Bioinformatics 1998, 14(1):68-73.

doi: $10.1186 / 1471-2180-10-183$

Cite this article as: Ong et al., Molecular analysis of type 3 fimbrial genes from Escherichia coli, Klebsiella and Citrobacter species BMC Microbiology 2010, 10:183

\section{Submit your next manuscript to BioMed Central} and take full advantage of:

- Convenient online submission

- Thorough peer review

- No space constraints or color figure charges

- Immediate publication on acceptance

- Inclusion in PubMed, CAS, Scopus and Google Scholar

- Research which is freely available for redistribution
C Biomed Central 